\title{
Image Processing Methods Usable for Object Detection on the Chessboard
}

\author{
Ladislav Beran ${ }^{\mathrm{a}}$, Pavel Chmelar and Lubos Rejfek \\ Department of Electrical Engineering, Faculty of Electrical Engineering and Informatics, University of Pardubice,Pardubice, Czech Republic
}

\begin{abstract}
Image segmentation and object detection is challenging problem in many research. Although many algorithms for image segmentation have been invented, there is no simple algorithm for image segmentation and object detection. Our research is based on combination of several methods for object detection. The first method suitable for image segmentation and object detection is colour detection. This method is very simply, but there is problem with different colours. For this method it is necessary to have precisely determined colour of segmented object before all calculations. In many cases it is necessary to determine this colour manually. Alternative simply method is method based on background removal. This method is based on difference between reference image and detected image. In this paper several methods suitable for object detection are described. Thisresearch is focused on coloured object detection on chessboard. The results from this research with fusion of neural networks for usercomputer game checkers will be applied.
\end{abstract}

\section{Introduction}

Image segmentation is the process of dividing pixels in an image into categories based on shared visual characteristics like a shared edge, colour, texture etc. The image segmentation is an important first step in object detection. Although many algorithms for image segmentation have been invented, there is no simple algorithm usable for all cases of image segmentation and object detection. For this detection, it is necessary to use combination of several methods for example colour segmentation and background removal. In case of colour segmentation, it is necessary to have precisely determined colour of searched object. In many cases, it is necessary to determine colour manually. The next problem in image segmentation and object detection is an added noise to signal by sensors. This noise, in case of camera withpoor lighting of the scene, can be added. In this paper several method suitable for object detection on chess board are described.

\section{Usable methods for detection}

\subsection{Background removal}

The background removal method is very simply method for object detection. This method is based on deduction of frame without any object from frame with object. The result is areas with segmented object. For using of this method, it is necessary to use an object with different color than background. This method is mathematically very simply and algorithm is described using equation (1), (2) and (3). The equation (1) describes a case when object is fitted on black squares (brighter object than background) and equation (2) describes case, when object is fitted on white squares (darker object than background).

$$
\begin{aligned}
& I[F(n)]=I[I(n)]-I[B G] \\
& I[F(n)]=I[B G]-I[I(n)]
\end{aligned}
$$

where: $\mathrm{I}[F(n)]$ is an image without background, $I[I(n)]$ is an captured image by camera, $I[B G]$ is first captured image - background.

An alternative of this method is possible to use an image difference. For this case alternative equation is used (3)

$$
|\mathrm{I}[\mathrm{F}(\mathrm{n})]-\mathrm{I}[\mathrm{F}(\mathrm{n}-1)]|>\operatorname{Tr}
$$

where: $I[F(n-1)]$ is a previous captured image, $\operatorname{Tr}$ is user selected threshold.

The main problem in case of background removal applied to chessboard is necessary to have pair of images. The first image is for case, when the object is fitted on black squares, the second for case, when object is fitted on white squares. It is not possible use universal algorithm, because when object is fitted on white squares, the maximal value will be less than 255 in case of 8 bit colour channels. If first algorithm is used, the object will be lost in saturation. For this case, it is necessary to use a subtraction captured image from template. The

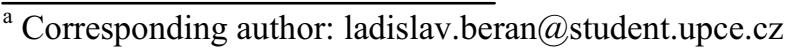


background removal method and its implementation are described in detail in 1 .

\subsection{Colour segmentation}

The colour segmentation is based on extraction similar shared visual characteristic - colour. The several types of colour spaces exist in the world. The main colour space is called RGB space. This space is based on red, green and blue component. Every from these component partially carry an information about brightness. Also, RGB is suitable for colour LCD display, but very bad for colour segmentation and analysis because of the high correlation among the R, G, and B components. From this case, it is necessary to use different colour space. In our case, we are using a HSV colour space. The conversion from RGB to HSV model is provided using equations (4) to (10).

$$
\mathrm{R}_{\mathrm{n}}=\frac{\mathrm{R}}{2^{\mathrm{b}}} ; \mathrm{G}_{\mathrm{n}}=\frac{\mathrm{G}}{2^{\mathrm{b}}} ; \mathrm{B}_{\mathrm{n}}=\frac{\mathrm{B}}{2^{\mathrm{b}}}
$$

where $R_{n}, G_{\mathrm{n}}$ and $B_{n}$ are normalized RGB components (Result is in range from 0 to 1 ), the $b$ is a bit length of each colour component.

$$
\begin{gathered}
\mathrm{C}_{\text {max }}=\max \left(\mathrm{R}_{\mathrm{n}}, \mathrm{G}_{\mathrm{n}}, \mathrm{B}_{\mathrm{n}}\right) \\
\mathrm{C}_{\text {min }}=\min \left(\mathrm{R}_{\mathrm{n}}, \mathrm{G}_{\mathrm{n}}, \mathrm{B}_{\mathrm{n}}\right) \\
\Delta=\mathrm{C}_{\text {max }}-\mathrm{C}_{\min }
\end{gathered}
$$

where $C_{\max }$ is maximal value from normalised RGB components, $C_{\min }$ is minimal value from RGB normalises components

$$
\text { Hue }=\left\{\begin{array}{cc}
0^{\circ} & \text { if } \Delta=0 \\
60^{\circ} \cdot\left(\frac{\mathrm{G}_{\mathrm{n}}-\mathrm{B}_{\mathrm{n}}}{\Delta} \bmod 6\right) & \text { ifC } \mathrm{max}_{\max }=\mathrm{R}_{\mathrm{n}} \\
60^{\circ} \cdot\left(\frac{\mathrm{B}_{\mathrm{n}}-\mathrm{R}_{-} \mathrm{n}}{\Delta}+2\right) & \text { ifC }_{\max }=\mathrm{G}_{\mathrm{n}} \\
60^{\circ} \cdot\left(\frac{\mathrm{R}_{\mathrm{n}}-\mathrm{G}_{\mathrm{n}}}{\Delta}+4\right) & \text { ifC }_{\max }=\mathrm{B}_{\mathrm{n}}
\end{array}\right\}
$$

$$
\text { Saturation }=\left\{\begin{array}{cl}
0 & \text { ifC }_{\max }=0 \\
\frac{\Delta}{C_{\max }} & \text { ifC }_{\max } \neq 0
\end{array}\right\}
$$

$$
\text { Value }=\mathrm{C}_{\max }
$$

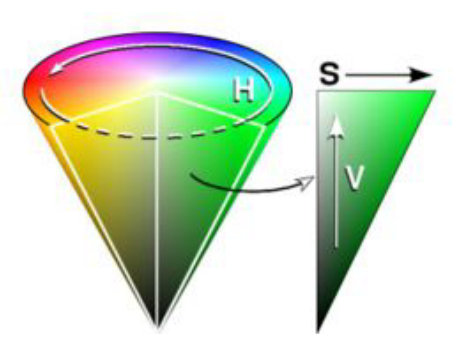

Figure 1. HSV color space.

The main advantage of HSV over the RGB is separated colours information. This information is called Hue, Saturation and Value 2, 3.

\section{Proposed approach}

\subsection{Background removal}

In our research, background removal algorithm was tested first. The background removal flowchart is shown at Figure 2.

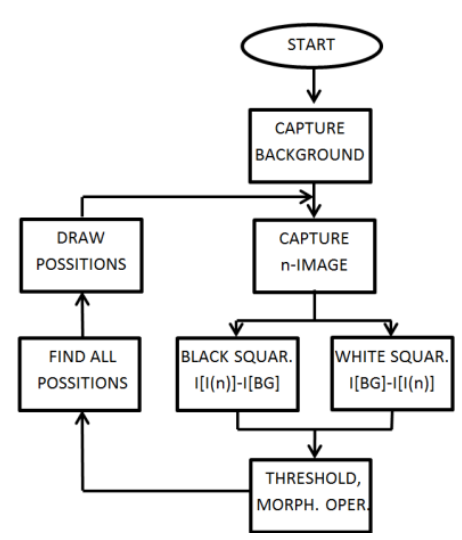

Figure 2. The background removal flowchart.

At first, algorithm capture template which is used as reference scene. After this process, algorithm starts periodically capturing the scene. The segmented object using the equations (1) and (2) is calculated and results are shown at Figure 3. At Figure 3 left side, segmented black squares is shown, at right side, segmented white squares is shown.
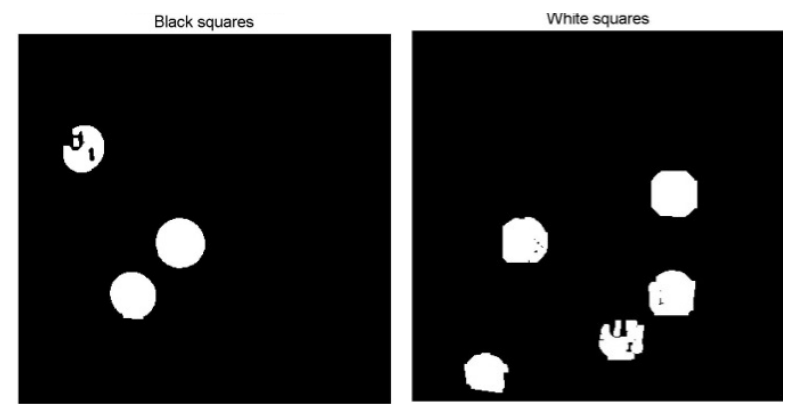

Figure 3. Segmented objects: black squares (left), white squares (right).

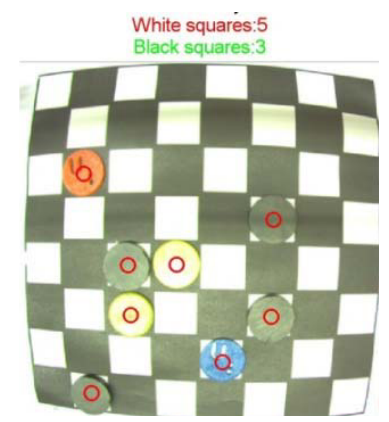

Figure 4. Final view of segmented and detected objects.

In this method, it is the main problem in case of wrongly selected threshold. Also, threshold can be selected correctly, but if lighting scenes can have small changes, which can cause totally wrong results. The next problem in background removal method is problem with similar colors - object and square. In this case, the object 
is lost in the background and detection is not possible. The example of this problem is shown at Figure 5 and Figure 6.
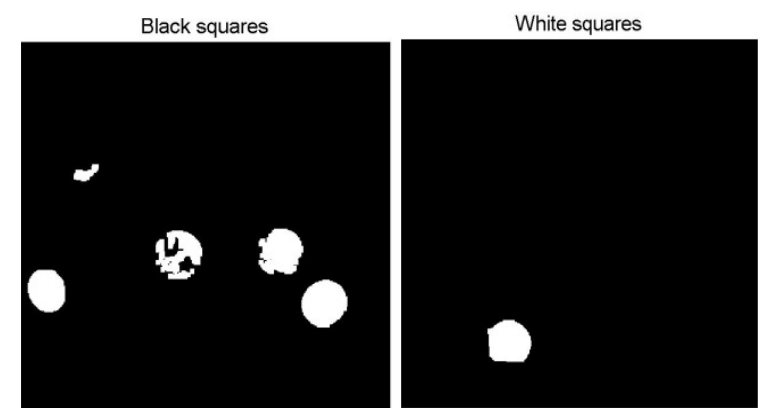

Figure 5. Wrongly segmented objects.

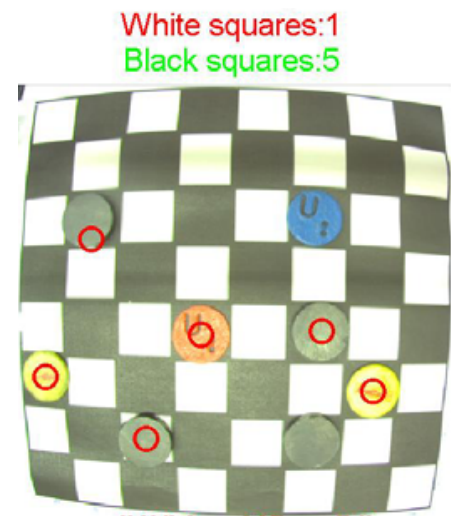

Figure 6. Wrongly segmented objects.

At Figure 6 the wrongly segmented object is shown. In case of grey object (undetected object in the lower part of image), the colour is mostly identic and algorithms are not able to detect this object. In case of blue object (undetected object on the top of image), is also problem with colour and hue but for this case, it is necessary to use extended algorithm for object detection like as circular object detection. Possible algorithms are described in the next chapters and in papers $4,5,6$.

\subsection{Colour segmentation and object detection}

The colour detection is based on detection an object with same/similar colour in the image. The models used during all word are RGB and HSV colour model. The RGB model is mainly used for displays. This colour model is based on Red, Green and Blue component. Every from component partially carry an information about brightness. Also, RGB is suitable for colour display, but very bad for colour scene segmentation and analysis because of the high correlation among the R, G, and B components. The result of colour segmentation is shown at Figure 7.

Using the results shown at Figure 7, the disadvantage of RGB colour model is possible to read. For case of red colour is possible to found maximal values (on red channel) which is shown in this cannel as a white colour, but additional information about colour is also written in another component. This colour model can be used only for strictly red, green and blue colours. For this case it is necessary to use different colour model called HSV Hue, Saturation and Value. This colour model has very big advantage comparison with RGB model - the colour component is not correlated. The RGB colour model for object detection with fusion of depth map in 7 was used.
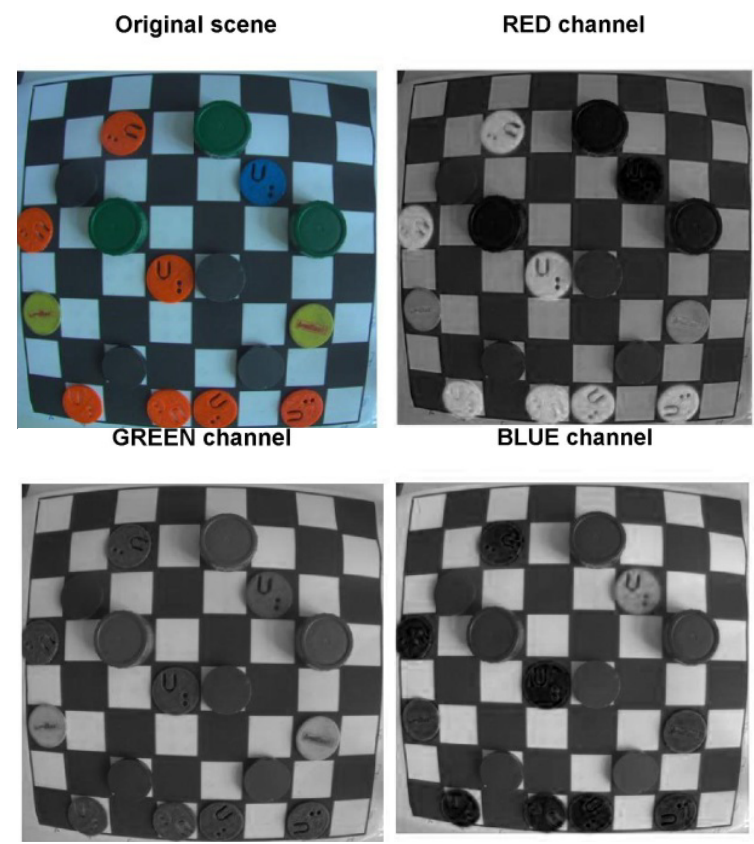

Figure 7. Example of RGB color channels.
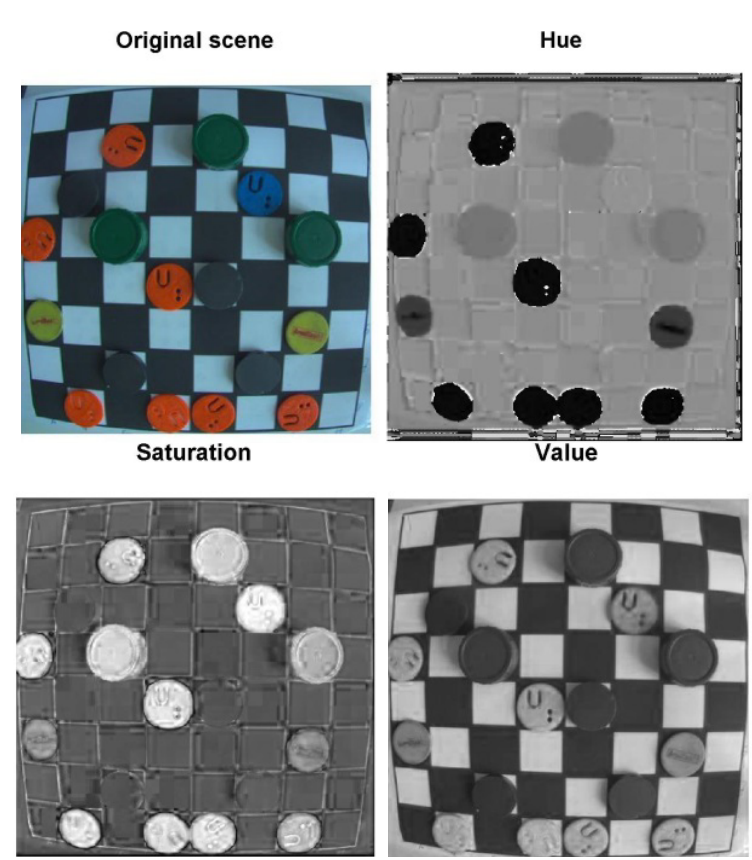

Figure 8. HSV color model.

At Figure 8 main difference with comparison RGB color model shown at Figure 7 is possible to shown. In case of HSV, the all information about colour in hue and saturation channel is written. The information from these channels are shown at Figure 9. This colour model is suitable for colour detection of objects. Also, from Figure 7 and Figure 8 it is possible to recognize problem 
with grey objects on a chessboard. These object is not possible to found using every known image processing methods, for case, when these object are fitted on the black squares. For our purpose it is neccary to use colours diiferent from black or white colours like a yellow, red, green, etc.

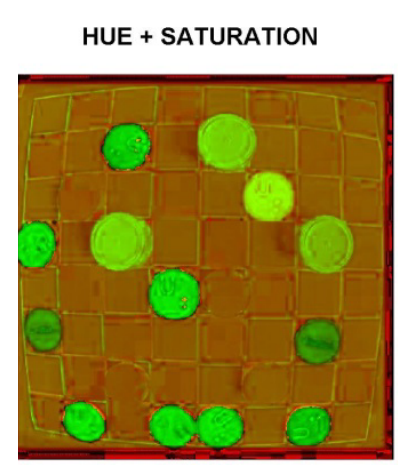

Figure 9. Hue and Saturation channel.

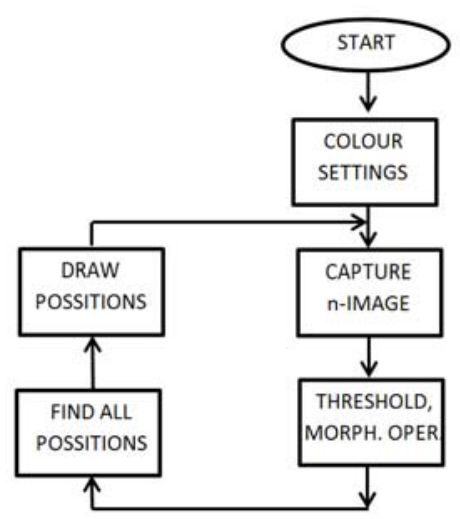

Figure 10. The color detection flowchart.

At Figure 10, the colour detection flowchart is shown. The algorithm after start capture scene which contain colour of the object which will be searched using algorithm. This sample is located in defined position. From this defined areas are calculated statistical values like average and standard deviation for all colour channels. These statistical values are calculated using equations (11) and (12). After this process, algorithm periodically captures a scene, calculate a threshold using statistical values, find all position of object and draw these positions to the original image. The colour segmentation algorithm is described using equation (13).

$$
\begin{gathered}
\overline{\mathrm{x}}=\frac{1}{\mathrm{~N}} \sum_{\mathrm{i}=1}^{\mathrm{N}} \mathrm{x}_{\mathrm{i}} \\
\sigma=\sqrt{\frac{1}{\mathrm{~N}} \sum_{\mathrm{i}=1}^{\mathrm{N}}\left(\mathrm{x}_{\mathrm{i}}-\overline{\mathrm{x}}\right)^{2}} \\
\left\{\begin{array}{cr}
\mathrm{x}=1 \text { when: } & \mathrm{x}_{\mathrm{HUE}} \in \overline{\mathrm{x}}_{\mathrm{HUE}} \pm \sigma_{\mathrm{HUE}} \\
\cap & \mathrm{x}_{\mathrm{SAT}} \in \overline{\mathrm{x}}_{\mathrm{SAT}} \pm \sigma_{\mathrm{SAT}} \\
\mathrm{x}=0 & \text { Otherwise }
\end{array}\right\}
\end{gathered}
$$

where $x$ is a pixel value in scene on defined position, $x_{H U E}$ and $x_{S A T}$ are a Hue/Saturation values of a pixel $\mathrm{x}$,
$\bar{x}_{H U E}$ and $\bar{x}_{S A T}$ are an average Hue/Saturation values calculated at start of the algorithm from the template, $\sigma_{H U E}$ and $\sigma_{S A T}$ are a standard deviations calculated from the template.
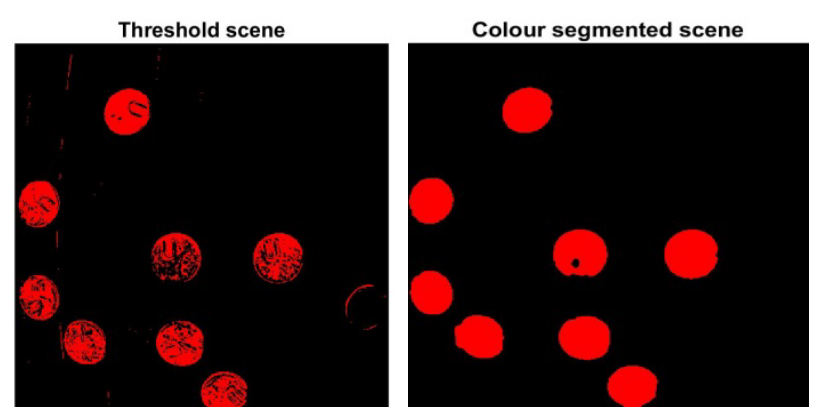

Figure 11. Threshold (left) and segmented (right) scene - Red objects.

At the Figure 11, threshold and segmented scene is shown.

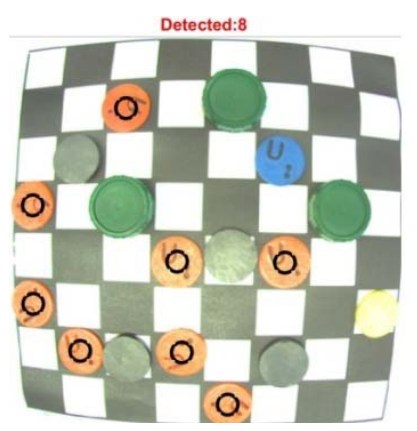

Figure 12. Detected red objects in the scene.

\section{Results and discussion}

At the Figure 13 our testing platform is shown. This platform is based on Basler acA-2500-14uc USB3 camera.

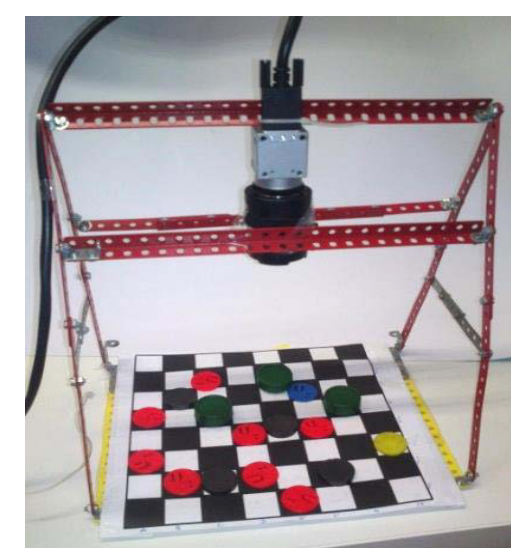

Figure 13. Testing platform.

In our research a several settings was tested for each method. During our testing, several advantages and disadvantages of suitable methods were found. These parameters of suitable methods are written in the Table 1 and Table 2. 
Table 1. Background removal parameters.

\begin{tabular}{|l|l|}
\hline \multicolumn{3}{|l}{ Background removal } \\
\hline \multirow{4}{*}{ Advantages } & Easy implementation \\
\cline { 2 - 2 } & Fast calculation \\
\cline { 2 - 2 } & Colour independence \\
\hline \multirow{2}{*}{ Disadvantages } & Need template before calculations \\
\cline { 2 - 2 } & Totally out when template is moved \\
\hline
\end{tabular}

Table 2. Color segmentation parameters.

\begin{tabular}{|l|l|}
\hline Colour segmentation \\
\hline \multirow{4}{*}{ Advantages } & Easy implementation \\
\cline { 2 - 2 } & Colour selection \\
\hline \multirow{4}{*}{ Disadvantages } & Problems with lighting of scene \\
\cline { 2 - 2 } & Need colour template before calculations \\
\cline { 2 - 2 } & Convert RGB -> HSV colour model \\
\cline { 2 - 2 } & Higher time consumption \\
\hline
\end{tabular}

Table 3. Time consumption of both methods.

\begin{tabular}{|c|c|}
\hline Method & $\begin{array}{c}\text { Average Time } \\
\text { consumption (s) }\end{array}$ \\
\hline Background removal & 0.14 \\
\hline Colour segmentation & 0.45 \\
\hline
\end{tabular}

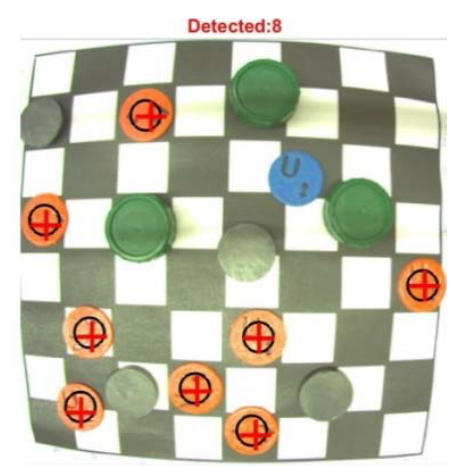

Figure 14. Detected red objects using background removal and color detection.

At Figure 14 the detected red objects using colour detection and background removal are shown. The detected centres using background removal using a red cross and black circles marks centres detected using colour segmentation are shown. The results are synthesis of detected centre using both methods. When calculated centres are located in defined interval between each detected centre, this point is shown in the result scene.

\section{Conclusion}

In this paper, methods for detection object placed on the chessboard are described. In this paper method of background removal was described. This method has satisfactory results and this method will be applied to the final system. The method of background removal is very sensitive for correctly selected threshold. The alone method detects all types of objection shapes and find a centroid of these object. For our purpose it is necessary to implement method of detection circular objects. Also, in this paper method based on color segmentation was described. In this method is necessary to know all parameters of colour - hue, value and saturation. These parameters from template are calculated. This method is based on detection specific colour and without change this method is not possible to detect another colour using one setting. At present, the colour of the object is selected manually. In this project will be defined two colours for players -for example green and red. In future, algorithm after start will be calibrated using special template with fitted game coins. In the future, algorithm from defined places records colour of game coins and if the colours will be different, game starts. For trouble-free game playing, it will be necessary to implement fusion of described methods - background removal and colour segmentation. The final research will be constructed as fusion of background removal, colour segmentation and circuit detection. These methods are implemented yet, but it is necessary to implement these methods with circular detection. This method is based on circular Hough transformation. At present, the both methods are implemented as complementary system, when if both detected centres are similar (in defined range), the object is marked as detected. In the future this rule will be modified. The object will be marked in case, if minimal two from three methods correctly detect centre of object. The results from this research will be applied to the usercomputer game called checkers.

\section{Acknowledgment}

The research was supported by the Internal Grant Agency of University of Pardubice, the project No. SGSFEI_2016_022.

\section{References}

1. P. Chmelar; A. Benkrid, "Efficiency of HSV over RGB Gaussian Mixture Model for fire detection," in Radioelektronika 2014 24th International Conference, 24, 1-4 (2014)

2. S. Sural; Qian Gang; S. Pramanik., "Segmentation and histogram generation using the HSV color space for image retrieval," in Image Processing-. Proceedings. 2002 International Conference on, 2, II589-II-592 (2002)

3. P. Chmelar, L. Beran, N. Kudriavtseva, The Laser Color Detection for 3D Range Scanning Using Gaussian Mixture Model, 25th International Conference Radioelektronika 2015, 25, 248-253 (2015)

4. W.E.L Grimson and D.P. Huttenlocher, On the sensitivity of the Hough transform for object recognition. IEEE Transactions on Pattern Analysis and Machine Intelligence, 12, 3. 255-274 (1990)

5. S. Umer; B.C. Dhara; B. Chanda, "A Fast and Robust Method for Iris Localization," in Emerging Applications of Information Technology (EAIT), 
2014 Fourth International Conference of, 4, 262-267 (2014)

6. M. Smereka; I. Duleba, Circular object detection using a modified hough transform, Int. J. Appl. Math. Comput. Sci. 18, 1 85-91 (2008)
7. P.Jetensky, S. Karamazov - Accessible Chessboard For Blind That Is Mouse And Keyboard Free. In International Conference on Applied Electrical Engineering and Informatics (AEI 2012), 72-76 (2012) 